\title{
Imaging and Spectroscopy of Graphene/Hexagonal Boron Nitride Lateral Heterostructure Interfaces
}

\author{
Leonardo Basile ${ }^{1,2}$, Lei Liu ${ }^{3}$, Gong $\mathrm{Gu}^{3}$ and Juan-Carlos Idrobo ${ }^{2}$ \\ ${ }^{1 .}$ Departamento de Fisica, Escuela Politécnica Nacional, Quito, Ecuador. \\ ${ }^{2}$ Center for Nanophase Materials Sciences, Oak Ridge National Laboratory, Oak Ridge, USA. \\ ${ }^{3 .}$ Department of EECS, the University of Tennessee, Knoxville, USA.
}

Recently, we demonstrated heteroepiaxy in two-dimensional space with a 1D sharp interface in a prototypical material system: graphene and hexagonal boron nitride (BN) [1]. Using microscopy probes on samples staying on the growth substrate, we established lattice coherence between the graphene seed and the $\mathrm{BN}$ monolayer grown from the edge of the seed. Atomic-resolution, element-contrast (Z-contrast) scanning transmission electron microscope (STEM) imaging of the boundary is desirable to reveal the presumably atomically sharp graphene-BN interface, but is not easily obtainable because contaminations introduced during the transfer process preferably reside over boundaries.

In this study, we examined the contaminant-covered graphene-BN boundary using electron energy-loss spectroscopy (EELS) in an aberration-corrected STEM, a Nion UltraSTEM ${ }^{\mathrm{TM}} 100$, equipped with a cold field emission electron source, a corrector of third and fifth order aberrations, and a Gatan Enfina spectrometer [2]. The microscope was operated at $60 \mathrm{kV}$ acceleration voltage to avoid knock-on damage of the graphene and BN lattices. A semi-convergence angle of $30 \mathrm{mrad}$, and 54 to $200 \mathrm{mrad}$ semi-collection angles were used to obtain the medium angle annular dark field (MAADF) images. The EEL spectrum map were collected with an energy resolution of $\sim 350 \mathrm{meV}$ and with an energy dispersion of $40 \mathrm{meV} /$ pixel.

Figure 1 (A) shows an experimental MAADF image of the graphene-BN boundary. Fast Fourier Transform of the areas shown in (A) indicates that the $\mathrm{BN}$ is aligned with the graphene monolayer. Evidence of a sharp interface is provided by the chemical map shown in (E), where the boron K-edge clearly defines a sharp graphene-BN interface, establishing EELS as a powerful "see-through" tool.

Fig 2 shows intensity profiles along the yellows lines of Fig 1. The left panel of Fig 2 shows that the graphene-BN boundary is composed of monolayer graphene and monolayer BN. The right panel shows a transition width of $0.5 \mathrm{~nm}$ between graphene and $\mathrm{BN}$ as determined from the boron K-edge signal.

A direct observation of the boundary at atomic resolution requires a reliable method to free the graphene-BN interface of contaminants. We will also discuss our effort to clean the buried interface by in-situ annealing. This method produces regions of thousands of nanometer squares of clean graphene and could be applied to other two dimensional (2D) materials.

\section{References:}

[1] L Liu et al, Science 343 (2014), p. 163.

[2] OL Krivanek et al, Ultramicroscopy 108 (2008), p. 179.

[3] This work was partially supported by the National Secretariat of Higher Education, Science, Technology and Innovation of Ecuador (SENESCYT) (LB), NSF (ECCS-1231808) and the Defense Advanced Research Projects Agency (approved for public release; distribution is unlimited) (LL \& GG). 
A portion of this research was conducted at the Center for Nanophase Materials Sciences (CNMS), which is sponsored at Oak Ridge National Laboratory by the Scientific User Facilities Division, Office of Basic Energy Sciences, U. S. Department of Energy (JCI).
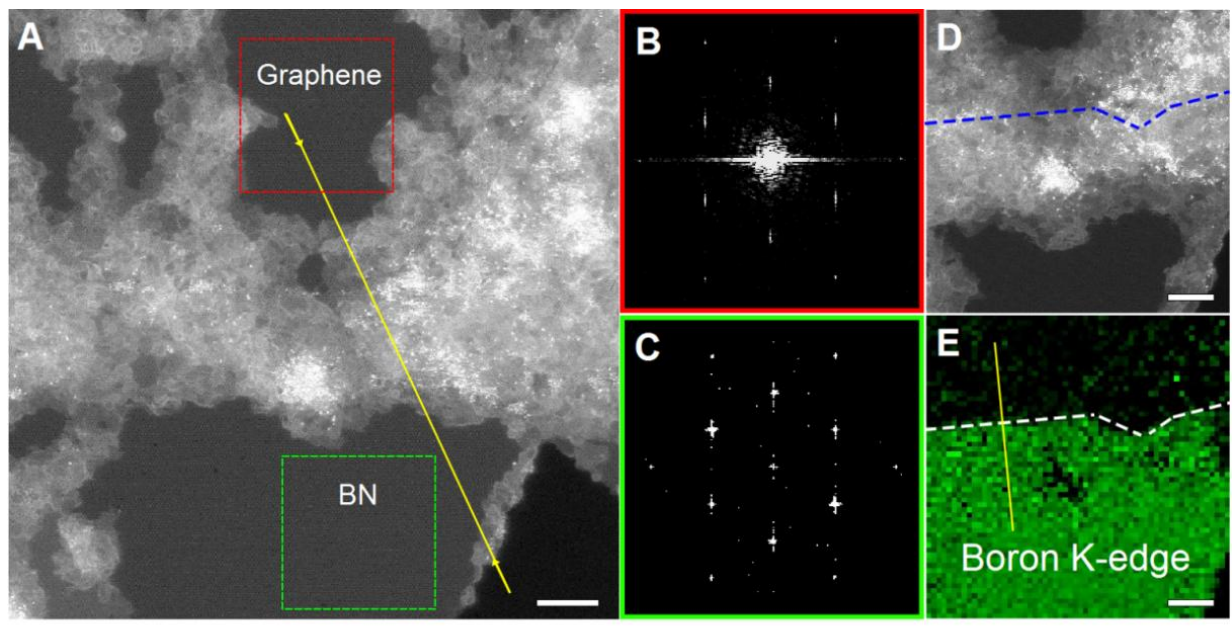

Figure 1. STEM-MAADF image and EELS. (A) MAADF image of a graphene-BN boundary. (B) y (C) fast Fourier transforms (FFT) of the highlighted areas shown in (A). (D) and (E) simultaneously acquired STEM image and electron energy loss spectrum map (boron K-edge) of a region contanining a buried graphene-BN boundary, respectively. The blue/white dashed lines indicate the boundary between the graphene and the BN. Scale bars are 5nm. Profiles in Fig. 2 are taken along yellow lines in (A) and (E). Adapted from Ref. [1].
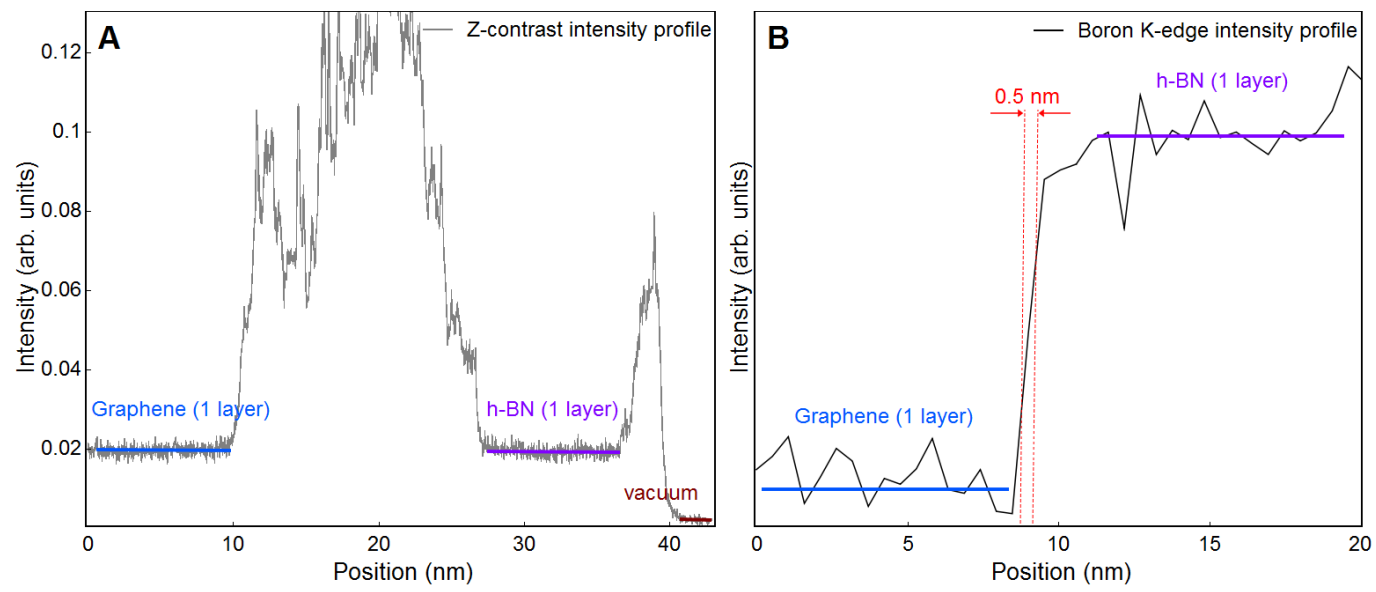

Figure 2. Intensity and EELS profiles. (A) Intensity profile along the yellow line in Fig. 1. The intensity profile indicates that the boundary is composed of monolayer graphene and BN. The intensity profile was obtained by integrating the signal across the interface within a width of $1.2 \mathrm{~nm}$. (B) Boron K-edge intensity profile along the yellow line in Fig. 1. The boundary is sharp with a transition width of $0.5 \mathrm{~nm}$. The transition width is defined from $25 \%$ to $75 \%$ of the values of the boron K-edge signal across the graphene-BN interface. The intensity profile was obtained by integrating the signal across the interface within a width of $4.8 \mathrm{~nm}$. The spatial resolution is $0.5 \mathrm{~nm}$. Adapted from Ref. [1]. 